performance in these areas. Bunge and colleagues from St. Louis presented arguments at the meeting that this type of lesion could best be explained by an abnormality of extracellular matrix (ECM) within the nerve, i.e. a deficiency in the microenvironment of the Schwann cell. This contention derives from evidence that Schwann cell development is retarded if only axonal contact is available without simultaneous contact with appropriate ECM material (Bunge \& Bunge J. Cell Biol. 78, 943; 1978).

Centre stage was reserved for a relatively new mutant, shiverer (the mutant $m l d$ may be an allele). Interest in this mutant derives from the specific lack of one of the major myelin componets - basic protein (Dupouey et al. Neurosci. Letters 12, 113; 1979). This constituent is of special interest for it causes a severe and sometimes fatal immune response when presented as an antigen with Freunds adjuvant. It previously had been assigned a quite specific role in normal myelin structure that of stabilizing the compacted membranes of the myelin sheath. It was therefore not unexpected that CNS myelination was found to be severely deficient in this mutant (Privat et al. Neurosci. Letters 12, 107; 1979) but it was surprising that myelin within the PNS, which also lacks basic protein, was quite normal in appearance, even at the level of the electron microscope (Kirschner \& Ganser Nature 283, 207; 1980). This mutant was much discussed at the Seillac meeting; several attendees suggested that available data indicate a different role for basic protein in CNS and PNS myelin.

\title{
Timing in embryological development
}

from Michael H.L. Snow \& Patrick P.L. Tam

NORMAL tables of embryonic development give the impression that the developmental processes are neatly co-ordinated, both spatially and temporally. The precision of timing of events is much stressed and frequently quoted in hours and minutes elapsed since the initiation of development. Certainly the synchrony observed between embryos of one species growing in a defined environment seems much more than mere coincidence. Evidence is accumulating that suggests a timing mechanism operates to control the emergence of developmental events. It is envisaged as a clock consisting of five components; the driving force (mainspring), operating an oscillatory mechanism (pendulum, balance wheel), which produces periodicity of emergent events (chimes on the hour), and all depending upon a linkage mechanism between oscillator and event (cog-wheels), and a capacity for adjustment in response to disturbance (correction for fast or slow running), (Brady Biological Clocks, Arnold, 1979).

In biological terms little is known about the driving force, the linkages or the homeostatic mechanisms but they can be presumed to exist. Studies have focussed on oscillators and the periodic events. More often than not the oscillatory mechanism has been ascribed to events in the cell cycle, DNA replication, cytokinesis. The premise that these reflect the operation of a clock has arisen from the characteristic regularity of the process in different tissues, and cell types. However the distinction made between proliferative

Michael H. L. Snow \& Patrick P. L. Tam. are in the MRC Mammalian Development Unit, University College, London. cycles, which merely increase the number of identical cells, and quantal cell cycles, which generate daughters with developmental options different from the mother cell (Holtzer et al. Q. Rev. Biophys. 8, $523 ; 1975)$ suggests that differentiative events may be gated by a periodicity not necessarily associated with cell cycles. Recent work throws some light on this subject.

The cell nucleus is generally regarded as the control tower for cellular functions and it has been implicated in the timing of various developmental events. In Ascidian embryos the specific expression of the enzyme acetylcholinesterase in presumptive muscle cells of the neurula (Whittaker J. Embryol. exp. Morph. 55, 43; 1980) has been shown to be under a stringent control apparently directly related to the number of DNA replications undergone in the nucleus. The timing of its appearence is unaffected when cell or nuclear divisions are prevented by metabolic inhibitors (Satoh J. Embryol. exp. Morph 54, 131; 1979). In the mouse embryo, cavitation of the morula to form a blastocyst seems more plausibly related to nuclear events since it is not governed by chronological time, nor interfered with by manipulation of cell number or supression of cytokinesis (Smith \& McLaren J. Embryol. exp. Morph. 41, 79; 1977).

Nevertheless, the nucleus can be excluded from other closely timed developmental events. In the mouse the time of compaction of the morula prior to blastocyst formation is not altered by changes in cell number, cell division, nuclear: cytoplasmic ratio or inhibition of DNA replication (Johnson, Pratt \& Handyside in Cellular and Molecular
Aspects of Implantation, Plenum, 1980). Similarly in Xenopus eggs periodic changes in contractile activity in the cortex of the cell are observed in perfect phase with cleavage divisions but persist in the absence of actual cell division and also continue when the cell is deprived of its nucleus (Hara, Tydeman \& Kirschner Proc. natn. Acad. Sci. U.S.A. 77, 462; 1980). Cytoplasmic or membrane associated mechanisms need to be considered for the control of these events. At the molecular level in the mouse the timing of synthesis of 'tissuespecific' polypeptides is the same in normal or experimentally disturbed embryos (Johnson, Handyside \& Braude in Development in Mammals 2, (ed. Von Blerkom \& Brockway) North-Holland 1977; Devel. Biol. 44, 148; 1975).

It is an attractive idea that the future development of the embryo is timed by a clock set in motion at the very beginning of development but is this feasible? The control of somitogenesis in Amphibian embryos is a later event that is precisely timed, regular and is currently explained in terms of a clock model (Cooke 3rd. Symp. Brit. Soc. Devl. Biol. Cambridge University Press, 1977; Pearson \& Elsdale J. Embryol. exp. Morph. 51, 27; 1979; Elsdale \& Pearson J. Embryol. exp. Morph $53,245 ; 1979)$. In mammals the later molecular events concerning the in vitro appearence of amylase activity in pancreatic primordia (Wessels \& Cohen Devel. Biol. 15, 237; 1977; Spooner, Cohen \& Faubion Devel. Biol. 61, 119; 1977) and the onset of responsiveness to androgen by mouse mammary tissue (Kratochwil Devl Biol. 61, 358; 1977) seem to relate to chronological age rather than cell cycle parameters since the biochemical changes coincide with the timing expected in vivo despite the fact that tissue mass and cell number may be quite different from that in the embryo in utero.

It is unlikely however that the whole embryo is controlled by a single clock. Recent studies in the mouse in which embryos recover from a severe size reduction show that the neural ectoderm, the somatic tissues of somite and limb-bud, and the germ cell population recover according to different timetables and appear to be under separate intrinsic controls (Snow \& Tam Nature 279, 555; 1979). So, either the clocks can be changed independently at tissue or organ level by an exogenous insult or, during development when various cell populations are set aside each one becomes a mosaic of time zones. It is intriguing to speculate whether the obvious co-ordination of tissue interactions in normal embryogenesis arises as the result of the harmonics generated by signals of differing periodicity originating from the different time zones. Such a system is reminiscent of the phase-shift model for spatial and temporal organisation proposed by Goodwin and Cohen ( $J$. Theoret. Biol. 25, 49; 1979). 